\title{
NMP22: Does it have a predictive potential for recurrence and progression in non-muscle-invasive bladder cancer?
}

\author{
Wassim Kassouf, MD, CM, FRCSC
}

See related article on page 454

$\mathrm{T}$ he overall rate of recurrence for non-muscle invasive bladder cancer (NMIBC) is $60 \%$ to $70 \%$, and the rate of progression is $30 \%$ to $60 \%$ in patients with high-risk features. Transurethral resection of bladder tumour (TURBT) followed by BCG induction with maintenance is the standard treatment option for high-risk NMIBC. All patients are recommended to undergo a cystoscopy at 3 months following TURBT, as cystoscopic findings at 3 months has been shown to be a prognostic factor of recurrence and progression of disease. Although there is no consensus for surveillance strategies, one recommendation that is widely adopted is to perform a follow-up cystoscopy with urine cytology every 3 to 4 months for 2 years, then every 6 months for the next 2 years, then yearly thereafter in patients with high-risk NMIBC. Despite stringent surveillance, many patients progress to muscle or metastatic disease and miss the window of opportunity for cure by radical extirpation.

Recently, several studies have advocated immediate cystectomy in patients with high-risk NMIBC (particularly T1G3), as $30 \%$ to $50 \%$ will progress despite intravesical therapy. However, there remains a significant risk of overtreatment with this strategy subjecting the patients to unnecessary morbidity of radical surgery including erectile dysfunction. Efforts are needed to better evaluate patients with high-risk NMIBC to identify more precisely those patients who will progress to muscle invasion and counsel them towards an early cystectomy.

Several studies have demonstrated the effectiveness of nuclear matrix protein 22 (NMP22) as a diagnostic tool for detection and surveillance of bladder cancer. However, no study has adequately assessed the role of NMP22 in predicting recurrence or progression. Two conflicting results have been reported on the prediction of recurrences in patients with positive NMP22 and negative cystoscopic findings; both studies were based on a small number of patients and did not stratify patients according to stage and grade. ${ }^{1,2}$ Lau and colleagues demonstrated that NMP22 appears to have a predictive value for future tumour recurrence but did not predict for progression in high-risk NMIBC. ${ }^{3}$ Since NMP22 could not predict for progression of disease, it cannot be used as an adjunct tool for the recommendation of early cystectomy in this patient population where progression rates are elevated. Over the next decade, as more molecular markers are being further evaluated in a prospective manner, an increasing role of risk-adapted management based on tumour molecular profiling will become integrated in clinical decision-making, particularly for patients with T1G3 disease.

Interestingly, NPM22 results did predict for recurrence of disease. Low- and intermediate-risk NMIBC (e.g., multifocal low grade Ta lesions) are associated with high recurrence and low progression rates. In this setting, NMP22 results may play a role to evaluate whether less stringent surveillance protocols (that includes invasive procedures such as cystoscopy) can be implemented. However, since the current study ${ }^{3}$ included high-risk tumours only with a relatively small sample size, the NMP22 predictive potential would need to be tested in a larger sample of patients with lower-risk tumours as the presented findings may not be applicable in the lower-risk setting.

From the Division of Urology, McGill University Health Centre, Montréal, QC

Competing interests: None declared.

This paper has been peer-reviewed.

\section{References}

1. Friedrich MG, Hellstern A, Toma MI, et al. Are false-positive urine markers for the detection of bladder carcinoma really wrong or do they predict tumor recurrence? Eur Urol 2003;43:146-50.

2. Gupta N P, Sharma N, Kumar R. Nuclear matrix protein 22 as adjunct to urine cytology and cystoscopy in follow-up of superficial TCC of urinary bladder. Urology 2009;73:592-6.

3. Lau P, Chin JL, Pautler $S$, et al. NMP22 is predictive of recurrence in high-risk superficial bladder cancer patients. Can Urol Assoc J 2009;3:454-8.

Correspondence: Dr. Wassim Kassouf, Division of Urology, McGill University Health Centre, 1650 Cedar Avenue, Rm L8-315, Montréal, QC H3G 1A4; fax: 514-934-8297; wassim.kassouf@muhc.mcgill.ca 